manner in which cocain can be used as a test for paralysis of the sympathetic nerve, as a valuable quality. Information with regard to other drugs in this respect would be valuable.

It has been suggested to me that the stimulating action on the sympathetic nerve might prove to be a test of the power of a drug to act as a stimulant of the central nervous system, and that so it might become a test of the liability of a drug to become a drug of addiction, a valuable test should it be substantiated.

My own experience of cocain substitutes is as follows :-

I have already expressed my opinion of novocain for infiltration ; but have not used it for instillation. I have found holocain in 2 per cent. solution satisfactory for superficial anaesthesia by instillation, but it causes distinctly more smarting than cocain of similar strength; I have not used it for intraocular operations. I have used 2 per cent. and 4 per cent. stovain by instillation both for superficial anaesthesia and for cataract operations, it is more irritating than cocain, it gives a satisfactory superficial anaesthesia, but I have thought it unsatisfactory for cataract operations, and on two occasions have had to complete the operation with the aid of cocain. I have used butyn a few times in the form of gelatin lamellae and 2 per cent. drops for superficial anaesthesia only, the anaesthesia seemed satisfactory but smarting was rather marked.

I know of no certain case of addiction to any of these drugs as a result of their clinical use by myself or others, the most I can say of one case is that I suspect the cocain habit was contracted as a result of the use of cocain ointment for a fistula-in-ano.

\title{
THE RADIOGRAPHIC LOCALIZATION OF FOREIGN BODIES IN THE EYE
}

\author{
BY \\ Professor Dr. L. DE LiebermaNi \\ BUDAPEST
}

In a previous paper dealing with the localization of foreign bodies in the eye (Arch. $f$. Augenheilk., Vol. LXXVI, p. 1ri) I gave a detailed description of the Sweet method, a method then little known in Europe though, in my opinion, one of the best. Sinice then it has become more popular. In this paper I emphasized the importance of making the exposure as brief as possible in order to prevent any movement from the exact optical direction. The longer the exposure the greater the risk of the patient losing the fixation point by movement of the head or eye. I next 
pointed out that for this purpose it is necessary ; (1) to $4 \mathrm{~s}^{\prime}$ an apparatus of high power; (2) to use X-ray tubes suitable for such high currents; $(3)$ to work with very sensitive plates.

At a meeting of the Budapest Royal Medical Association on May 12, 1923, in the course of a discussion on this subject, I had an opportunity of offering a suggestion as to a means of compensating for a longer exposure when any of the three conditions enumerated above could not be fulfilled. I consider that under good conditions the time of exposure should never exceed three seconds in the straight and five seconds in the oblique position and even less when possible. The chief difficulty in obtaining such brief exposures was the poor quality of the Roentgen plates we had to use immediately after the war. There may, of course, also be difficulties with apparatus and tubes. To meet these difficulties I suggested a method of ascertaining. whether the eye had kept its exact position in the Sweet apparatus, and if not, a means of making the necessary correction without the necessity of making another exposure. 'This is of the greatest importance in all cases requiring absolute exactness in localization, especially in those cases in which the removal of non-magnetic bodies necessitates direct operation.

The method consists in attaching two metal marks to the eye before the exposure. These marks are two metal plates of about $2 \mathrm{~mm}$. diameter in which two holes have been drilled to allow of their being attached by sutures to the cocainized bulbar conjunctiva. They must be made of a non-magnetic metal in cases in which a magnet is to be used. The sutures are first tied in position with a good hold of the episcleral tissue and the two ends passed through the holes in the two plates and again tied over the plates. After the exposure has been made the upper knot is cut and the plates removed. The sutures remain attached to the conjunctiva by the lower knot to serve as a guide during the operation. Since the localization of the mark knots is exactly known, we can easily see if the optical fixation of the centre of the cornea has been maintained during the exposure. If this has not been the case the necessary correction can be made with great ease. If the situation of the foreign body is known approximately it is advisable to place the metal marks, or one of them, as near to this point as possible. If this is not the case they should be fixed close to the limbus in order easily to control the fixed centre point.

When I made this suggestion I was aware of a similar method described by Dr. Pischel of San Francisco at a meeting of the Ophthalmological Society of Vienna (Wien. Klin. Wochenschr., No, 28, p. 1337, 1923). Pischel uses metal needles, $3 \mathrm{~mm}$. long, inserted into the conjunctiva to get fixed points that allow an 
approximate localization and dispenses with the help of Sweet's or any other exact geometrical localizing apparatus.

I do not wish to claim any priority over Pischel for the suggestion of the fixing of metal marks to the eye as his communication was made at Vienna ten days before mine at Budapest, although I had not seen his paper at that time, but I wish to point out the following facts :

(1) Both Pischel's and my method are a distinct advance on the earlier device of Wessley who applied a wire cross upon the cornea, since this wire cross cannot be left in position up to the time of and during the operation as our conjunctival marks and thread knots can. The latter, therefore, allow of a more exact localization.

(2) Pischel's intention is to substitute his method for the use of the Sweet apparatus when this is unobtainable, and also for use in urgent cases in which he considers that the use of the Sweet apparatus causes too much loss of time. He admits that the mark method with simply a frontal and sagittal radiogram is not so exact as the Sweet localization, but considers that this is more than compensated for by the saving of time. He seems to assume that the Sweet localization can only be read after the plate has been dried.

I do not offer my method as a substitute for the Sweet or any other geometrical method of similar accuracy but as an adjuvant in the obtaining of still more accurate results in cases in which the greatest precision is required. On the other hand, I should like to emphasize what I pointed out in my former paper (loc. cit.), i.e. that the Sweet method does not involve any loss of time in urgent cases, as the points necessary for localization can be copied from the wet plate to a simple dry glass plate and transferred to the paper scheme with perfect accuracy. This can be done as soon as the plate has been removed from the fixing bath and got readv as soon as the patient can be prepared for operation.

(3) I consider that the method of fixing the metal marks with sutures is of distinct value, especially in cases of non-magnetic bodies, when it may be necessary to make the incision exactly at the marked point. The sutures can be firmly anchored in the episcleral tissue where they remain immovable and do not interfere in any way with the operation. The needles, on the other hand are liable to displacement both before and during the operation. If the sutures are too deeply inserted into the episcleral tissue their removal may present some difficulty, especially after an operation perforating the sclera. I presume that Pischel also leaves his needles in position during the operation, though this is not clearly stated in the printed record of the Vienna meeting. 